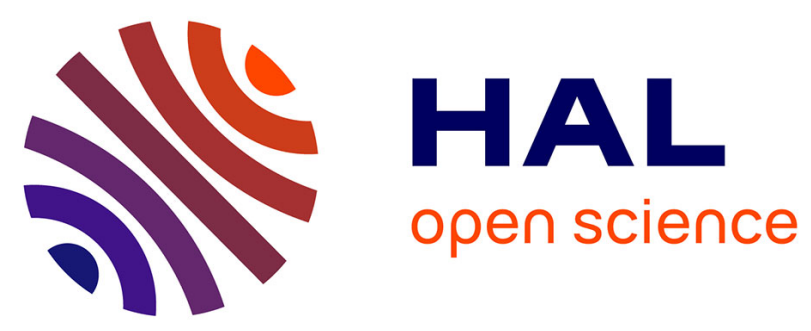

\title{
Improved "cure on demand" of aromatic bismaleimide with thiol triggered by retro-Diels-Alder reaction
}

Vincent Froidevaux, Mélanie Decostanzi, Abdelatif Manseri, Sylvain Caillol, Bernard Boutevin, Rémi Auvergne

\section{- To cite this version:}

Vincent Froidevaux, Mélanie Decostanzi, Abdelatif Manseri, Sylvain Caillol, Bernard Boutevin, et al.. Improved "cure on demand" of aromatic bismaleimide with thiol triggered by retro-Diels-Alder reaction. Frontiers of Chemical Science and Engineering, 2021, 15 (2), pp.330-339. 10.1007/s11705020-1929-6 . hal-03166843

\section{HAL Id: hal-03166843 \\ https://hal.science/hal-03166843}

Submitted on 11 Mar 2021

HAL is a multi-disciplinary open access archive for the deposit and dissemination of scientific research documents, whether they are published or not. The documents may come from teaching and research institutions in France or abroad, or from public or private research centers.
L'archive ouverte pluridisciplinaire HAL, est destinée au dépôt et à la diffusion de documents scientifiques de niveau recherche, publiés ou non, émanant des établissements d'enseignement et de recherche français ou étrangers, des laboratoires publics ou privés. 


\section{Improved "cure on demand" of aromatic bismaleimide with thiol triggered by retro-Diels-Alder reaction}

Vincent Froidevaux, Mélanie Decostanzi, Abdelatif Manseri, Sylvain Caillol, Bernard Boutevin, Rémi Auvergne (

Institut Charles Gerhardt, Montpellier, UMR 5253, CNRS, Université de Montpellier, Ecole Nationale Supérieure de Chimie de Montpellier, 34296 Montpellier, France

E-mail: remi.auvergne@enscm.fr

Abstract This study focuses on the synthesis of new liquid aromatic bismaleimide monomers in order to improve self-curing on demand (SCOD) systems previously based on aliphatic bismaleimides. These SCOD systems are based on Diels-Alder (DA)/retro-DA reactions. The syntheses of new different aromatic bismaleimides with ester and amide bonds are presented. These maleimides have been protected using DA reaction and characterized by ${ }^{1} \mathrm{H}$ NMR analysis to determine protection rate and diastereomer ratios. The retro-DA reactions of both aromatic and aliphatic DA adducts in presence of thiol molecules were studied. Kinetic analysis was monitored by ${ }^{1} \mathrm{H}$ NMR and compared to model study. Finally, both aromatic and aliphatic bismaleimides-based polymers were synthesized with 2-mercaptoethyl ether and thermal properties of polymers were compared. The glass transition temperature values ranged from $-20^{\circ} \mathrm{C}$ to $14^{\circ} \mathrm{C}$ and very good thermal stabilities were observed (up to $\left.300^{\circ} \mathrm{C}\right)$.

Keywords thiol-ene polymerization, self-curing on demand, thia-michael addition, Diels-Alder, maleimide

\section{Introduction}

Diels-Alder (DA) reactions are mainly used to prepare self-healing materials [1-3]. Temperature is the easier stimulus to break organic bonds between two molecules. Many well-known systems are capable of forming reversible covalent bonds at different temperatures [4-16]. However, the thermal reversibility can be used (and is used to a low extend) for other aims, such as the recycling of polymers or to protect reversible functional groups in order to control polymerization or crosslinking.

The concept of self-curing on demand (SCOD) is used to protect reactive functions and to control polymerization or cross-linking reactions. Most of existing SCOD systems are based on electromagnetic waves - such as ultraviolet light (UV), electron beam, magnetic radiation or heat. The use of UV is not favored since these radiations are harmful and in most of the cases, fillers which are present in the polymer, reduce the efficiency of UV. Indeed, radiation technologies present many advantages but are mostly limited to thin coating applications [17]. Furthermore, thermal SCOD was extensively developed for polyurethane chemistry. Indeed, the free isocyanate function can be protected by a blocking group containing a labile proton. The cleavage and release of blocking group, at high temperature, yields again an isocyanate function which can react and lead to crosslinking of polymer. Various blocking groups are used industrially or studied by research groups [18-25]. However, the blocking of isocyanate suffers from many limitations: the moisture sensitivity of isocyanate function, the high toxicity of free 
isocyanates and the general drawback common at all system, the undesirable release of volatile blocking agents.

In a previous model study [26] we have studied the DA reaction with different reactants and have shown that the retro-DA reaction can occur below $100^{\circ} \mathrm{C}$. More precisely, reverse-DA (rDA) starts at $60^{\circ} \mathrm{C}$ for the endo adduct. The endo adduct is always unblocked at lower temperature than the exo adduct, which is thermodynamically more stable [27]. We also showed that the retro-DA reaction could be accelerated by the Michael addition of a nucleophile (for instance a thiol) [28,29]. It was shown that the Michael addition occured in situ with the maleimide compound, which avoided the reprotection of the maleimide by DA reaction. In another paper, we have used a polyfunctional aliphatic maleimide to obtain a material through an original thermal SCOD system [30]. However, the crosslinking duration was $17 \mathrm{~h}$ at $110^{\circ} \mathrm{C}$. These long conditions can be explained by the presence of exo diastereoisomers which are totally unblocked at high temperature. In order to obtain better properties, aromatic maleimides were originally synthesized in the present study.

Few examples in literature deal with the synthesis of aromatic bismaleimides [31]. Hence, aromatic bismaleimides with a polybutylene glycol spacer [32] and an ester link or aromatic bismaleimides with flexible spacer as ethylene, diethylene or triethylene glycol and an ether or ester link to ameliorate the process without loss of thermal stability were reported and found application in adhesives formulation or electronics [33]. The group of Milano has developed maleimides with a higher aromatic density in order to increase the thermal properties of the obtained materials [34]. These new maleimides found applications in thermoset liquid crystals materials.

In our study, liquid maleimides are preferred over solid ones for an easier processing. Indeed, the use of liquid maleimides helps the homogeneity of the mixture during solvent-free reaction at room temperature. In that purpose, Huntsman Jeffamines ${ }^{\circledR}$ (ED-600 and ED-900) were used to synthesize amide-based aromatic bismaleimides whereas Pluronic ${ }^{\circledR}$ L35 and Terathane ${ }^{\circledR} 650$ diols were used to synthesize ester-based aromatic bismaleimides. Huntsman Jeffamines ${ }^{\circledR}$ are tri-block copolymers of poly(ethyleneoxide) (PEO) and poly(propyleneoxide) (PPO) (thus avoiding PEO crystallinity). Pluronic ${ }^{\circledR}$ L35 is also a tri-block copolymer of PEO and PPO whereas Terathane ${ }^{\circledR} 650$ is a poly(1,4-butanediol).

Then bismaleimides were synthesized therefrom and DA reactions were performed. The exo/endo ratio was studied at different temperatures and compared to the one obtained with aliphatic moiety. The retro-DA reactions were then studied and kinetics was monitored by ${ }^{1} \mathrm{H}$ NMR. Finally, polymers were synthesized and their thermal properties were compared.

\section{Experimental}

\subsection{Materials and methods}

Furfuryl acetate (98\%), maleic anhydride (99\%), $\mathrm{ZnBr}_{2}$ (98\%), hexamethyldisilazane (HMDS) (>99\%), anhydrous sodium sulfate, triethylamine (99\%), sodium acetate, $p$-aminobenzoic acid, $N, N$-dimethylformamide, thionyl chloride, glycerol ethoxylate, terathane ${ }^{\circledR} 650$ (poly(tetrahydrofuran)), tert-butylcatechol and thiophenol were purchased from Sigma-Aldrich. Various Jeffamines (ED-600 and ED-900) were given by Huntsman. Dichloromethane, methanol, benzene and tetrahydrofuran (THF) 
were purchased from Van Waters Rogers company. Pluronic $®$ L35 was purchased from Badische Anilin-\& Soda-Fabrik.

Proton and carbon nuclear magnetic resonance $\left({ }^{1} \mathrm{H}\right.$ and ${ }^{13} \mathrm{C}$ NMR) analyses were performed in $\mathrm{CDCl}_{3}$, DMSO- $d_{6}$ and $\left(\mathrm{CD}_{3}\right)_{2} \mathrm{CO}$ using a Bruker Avance $400 \mathrm{MHz}$ NMR spectrometer at a temperature of $25^{\circ} \mathrm{C}$. NMR samples were prepared as follows: $10 \mathrm{mg}$ of product for ${ }^{1} \mathrm{H}$ experiment in around $0.4 \mathrm{~mL}$ of deuterated solvent. The chemical shifts were reported in part per million relative to tetramethylsilane.

Thermogravimetric analyses (TGA) were performed using a TG 209F1 apparatus (Netzsch) at a heating rate of $10^{\circ} \mathrm{C} \cdot \mathrm{min}^{-1}$. Approximately $10 \mathrm{mg}$ of sample was placed in a platinum crucible and heated from room temperature to $700^{\circ} \mathrm{C}$ under nitrogen atmosphere $\left(60 \mathrm{~mL} \cdot \mathrm{min}^{-1}\right)$.

Differential scanning calorimetry analyses were carried out using a NETZSCH DSC200F3 calorimeter. Constant calibration was performed using indium, $n$-octadecane and $n$-octane standards. Nitrogen was used as the purge gas. Approximately $10 \mathrm{mg}$ of sample were placed in perforated aluminum pans and the thermal properties were recorded between $-150^{\circ} \mathrm{C}$ and $200^{\circ} \mathrm{C}$. The glass transition temperature $\left(T_{g}\right)$ values were measured during the second heating ramp to erase the thermal history of the polymer. All the reported temperatures are mean values.

\subsection{The syntheses of precursors, monomers, and polymers}

\subsubsection{Precursors syntheses}

Synthesis of 4-(2,5-dioxo-2,5-dihydro-1H-pyrrol-1-yl)benzoic acid 1. Maleic anhydride (1.1 eq/amine function) was reacted with $p$-aminobenzoic acid (1 eq) in DMF $\left(4 \mathrm{~mL} \cdot \mathrm{g}^{-1}\right)$ during $1 \mathrm{~h}$. The amine was added dropwise. After total addition, the temperature was raised to $70^{\circ} \mathrm{C}$ for $4 \mathrm{~h}$. Then the maleic acid formed was precipitated in cold water. The product was filtered off and was dried under oven at $80^{\circ} \mathrm{C}$ for one night. A yellow pale product was obtained (Yield $=98 \%$ ). This product was reacted with a mixture of acetic anhydride $\left(2 \mathrm{~mL} \cdot \mathrm{g}^{-1}\right.$ of acetic acid) and sodium acetate $(10 \mathrm{~mol}-\%)$ at $60^{\circ} \mathrm{C}$ for $4 \mathrm{~h}$. The mixture was put in a large amount of cold water to precipitate the 4-(2,5-dioxo-2,5-dihydro-1H-pyrrol-1-yl)benzoic acid. The product was filtered off and recrystallized in a methanol/water $(6 / 1)$ mixture. Then the product was dried at $80^{\circ} \mathrm{C}$ for one night. A yellow pale solid was obtained, yields 88\%. ${ }^{1} \mathrm{H}$ NMR (400 MHz, DMSO, $\left.\delta \mathrm{ppm}\right): 13.1\left(\mathrm{~s}, 1 \mathrm{H}, \mathrm{H}_{\mathrm{d}}\right), 8.07-8.03\left(\mathrm{~m}, 2 \mathrm{H}, \mathrm{H}_{\mathrm{c}}\right.$ ), 7.52-7.48 (m, 2H, $\left.\mathrm{H}_{\mathrm{b}}\right), 7.21\left(\mathrm{~s}, 2 \mathrm{H}, \mathrm{H}_{\mathrm{a}}\right) .{ }^{13} \mathrm{C}$ NMR (100 MHz, DMSO, $\left.\delta \mathrm{ppm}\right): 169.6\left(\mathrm{C}_{\mathrm{h}}\right), 166.7\left(\mathrm{C}_{\mathrm{e}}\right)$, $135.5\left(\mathrm{C}_{\mathrm{f}}\right), 134.9\left(\mathrm{C}_{\mathrm{a}}\right), 129.9\left(\mathrm{C}_{\mathrm{c}}\right), 129.6\left(\mathrm{C}_{\mathrm{g}}\right), 126.1\left(\mathrm{C}_{\mathrm{b}}\right)$.

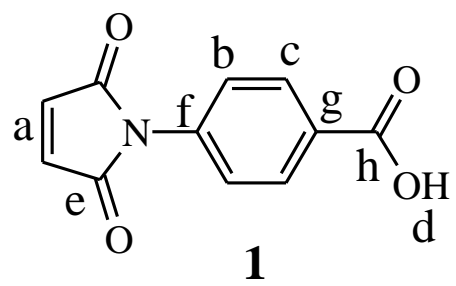

Synthesis of the 4-(2,5-dioxo-2,5-dihydro-1H-pyrrol-1-yl)benzoyl chloride 2. A mixture of 4-(2,5-dioxo-2,5-dihydro-1H-pyrrol-1-yl)benzoic acid (1 eq), of thionyl chloride (2.5 eq) and tert-butylcatechol $(0.01 \mathrm{eq})$ was refluxed for $3 \mathrm{~h}$. The thionyl chloride was added drop by drop. Unreacted thionyl chloride was evaporated under vacuum. The residual yellow solid was recrystallized in benzene and filtered off to obtain pure 4-(2,5-dioxo-2,5-dihydro-1H-pyrrol-1-yl)benzoyl chloride, yield 73\%. ${ }^{1} \mathrm{H}$ NMR (400 MHz, DMSO, $\delta$ ppm): 8.23-8.2 (m, 2H, $\mathrm{H}_{\mathrm{c}}$ ), 7.66-7.63 (m, 2H, $\mathrm{H}_{\mathrm{b}}$ ), 6.92 (s, 
2H, $\left.\mathrm{H}_{\mathrm{a}}\right) \cdot{ }^{13} \mathrm{C}$ NMR (100 MHz, DMSO, $\left.\delta \mathrm{ppm}\right): 168.5\left(\mathrm{C}_{\mathrm{d}}\right), 167.5\left(\mathrm{C}_{\mathrm{e}}\right), 137.6\left(\mathrm{C}_{\mathrm{f}}\right), 134.5\left(\mathrm{C}_{\mathrm{a}}\right), 132.2\left(\mathrm{C}_{\mathrm{c}}\right)$, $131.6\left(\mathrm{C}_{\mathrm{g}}\right), 125.1\left(\mathrm{C}_{\mathrm{b}}\right)$.<smiles>O=C(Cl)c1ccc(N2C(=O)C=CC2=O)cc1</smiles>

\subsubsection{Synthesis of aromatic maleimides derivatives}

A solution of 4-(2,5-dioxo-2,5-dihydro-1H-pyrrol-1-yl)benzoyl chloride (2.05 eq) in dichloromethane $\left(40 \mathrm{~mL} \cdot \mathrm{g}^{-1}\right)$, was added dropwise under nitrogen at room temperature, on a mixture of spacer $(1 \mathrm{eq})$ and triethylamine (2.1 eq). Then, the mixture was heated at $40^{\circ} \mathrm{C}$ for $5 \mathrm{~h}$. The mixture was filtered and extracted three times with salted water. The organic layer was dried over $\mathrm{Na}_{2} \mathrm{SO}_{4}$ and the solvent was evaporated under vacuum. The organic layer was dried over $\mathrm{Na}_{2} \mathrm{SO}_{4}$ and the solvent was evaporated under vacuum. In the case of the synthesis of BM Ar Terat650 and BM Ar L35 the product was solubilized in ether and the excess of acid chloride was filtered off. Then, the solvent was evaporated.

BM Ar L35 $(x+y=16$ and $z=21)$ (SI1). Orange viscous liquid, yield $56 \%$. ${ }^{1} \mathrm{H}$ NMR (400 MHz, $\left.\left(\mathrm{CD}_{3}\right)_{2} \mathrm{CO}, \delta \mathrm{ppm}\right): 8.14\left(\mathrm{~m}, 4 \mathrm{H}, \mathrm{H}_{\mathrm{c}}\right), 7.58\left(\mathrm{~m}, 4 \mathrm{H}, \mathrm{H}_{\mathrm{b}}\right), 7.1\left(\mathrm{~s}, 4 \mathrm{H}, \mathrm{H}_{\mathrm{a}}\right), 4.46\left(\mathrm{~m}, 4 \mathrm{H}, \mathrm{H}_{\mathrm{d}}\right), 3.84(\mathrm{~m}, 4 \mathrm{H}$, $\left.\mathrm{H}_{\mathrm{e}}\right)$, 3.67-3.47 (m, 156H, $\left.\mathrm{H}_{\mathrm{f}}, \mathrm{H}_{\mathrm{g}}, \mathrm{H}_{\mathrm{i}}, \mathrm{H}_{\mathrm{j}}\right), 1.12$ and $1.1\left(2 \mathrm{~s}, 63 \mathrm{H}, \mathrm{H}_{\mathrm{k}}\right)$.

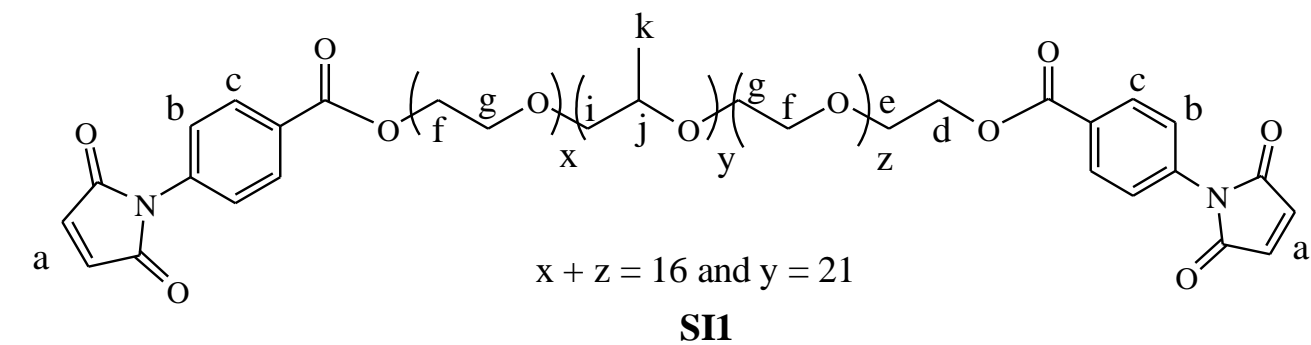

BM Ar ED-600 ( $x+y=3.6$ and $z=9)(\mathrm{SI} 2)$. Orange viscous liquid, yield 63\%. ${ }^{1} \mathrm{H}$ NMR $(400 \mathrm{MHz}$, $\left.\left(\mathrm{CD}_{3}\right)_{2} \mathrm{CO}, \delta \mathrm{ppm}\right): 7.98\left(\mathrm{~m}, 4 \mathrm{H}, \mathrm{H}_{\mathrm{c}}\right), 7.57\left(\mathrm{~s}_{\mathrm{br}}, 2 \mathrm{H}, \mathrm{H}_{\mathrm{d}}\right), 7.46\left(\mathrm{~m}, 4 \mathrm{H}, \mathrm{H}_{\mathrm{b}}\right), 7.06\left(\mathrm{~s}, 4 \mathrm{H}, \mathrm{H}_{\mathrm{a}}\right), 4.28(\mathrm{~s}, 2 \mathrm{H}$, $\left.\mathrm{H}_{\mathrm{e}}\right), 3.64-3.35\left(\mathrm{~m}, 48 \mathrm{H}, \mathrm{H}_{\mathrm{g}}, \mathrm{H}_{\mathrm{h}}, \mathrm{H}_{\mathrm{i}}, \mathrm{H}_{\mathrm{j}}\right), 1.26$ and $1.08\left(2 \mathrm{~m}, 23 \mathrm{H}, \mathrm{H}_{\mathrm{f}}\right)$.

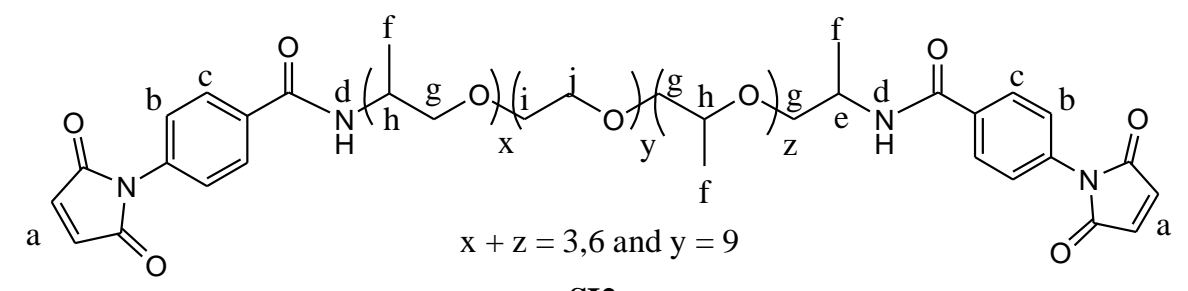

SI2

BM Ar ED-900 ( $x+y=6$ and $z=12,5)$ (SI3). Orange viscous liquid, yield 62\%. ${ }^{1} \mathrm{H}$ NMR $(400 \mathrm{MHz}$, $\left.\left(\mathrm{CD}_{3}\right)_{2} \mathrm{CO}, \delta \mathrm{ppm}\right): 7.99\left(\mathrm{~m}, 4 \mathrm{H}, \mathrm{H}_{\mathrm{c}}\right), 7.57\left(\mathrm{~s}_{\mathrm{br}}, 2 \mathrm{H}, \mathrm{H}_{\mathrm{d}}\right), 7.47\left(\mathrm{~m}, 4 \mathrm{H}, \mathrm{H}_{\mathrm{b}}\right), 7.07\left(\mathrm{~s}, 4 \mathrm{H}, \mathrm{H}_{\mathrm{a}}\right), 4.28(\mathrm{~s}, 2 \mathrm{H}$, $\left.\mathrm{H}_{\mathrm{e}}\right), 3.67-3.37\left(\mathrm{~m}, 76 \mathrm{H}, \mathrm{H}_{\mathrm{g}}, \mathrm{H}_{\mathrm{h}}, \mathrm{H}_{\mathrm{i}}, \mathrm{H}_{\mathrm{j}}\right), 1.25$ and $1.08\left(2 \mathrm{~m}, 16 \mathrm{H}, \mathrm{H}_{\mathrm{f}}\right)$. 


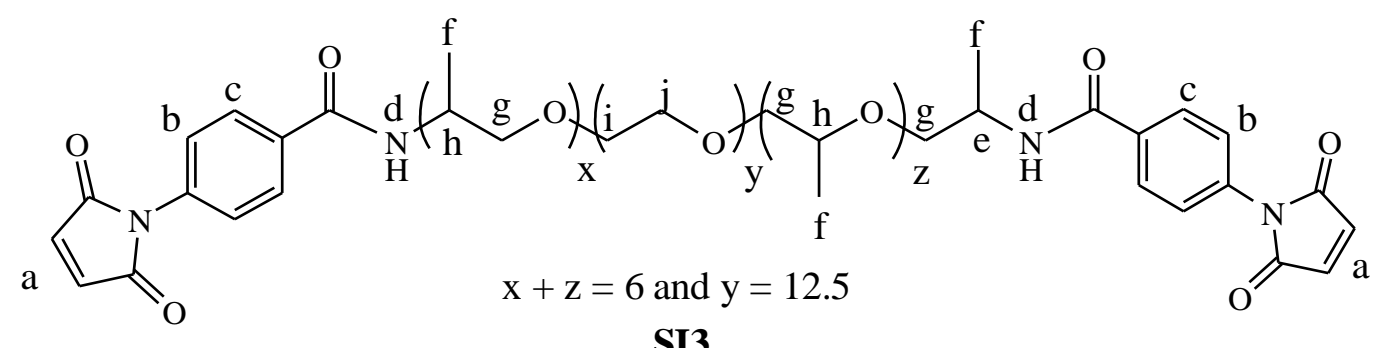

SI3

BM Ar Terat650 ( $y=8$ ) (SI4). Orange viscous liquid, yield 60\%. ${ }^{1} \mathrm{H}$ NMR $\left(400 \mathrm{MHz},\left(\mathrm{CD}_{3}\right)_{2} \mathrm{CO}, \delta\right.$ ppm): $8.12\left(\mathrm{~d}, 4 \mathrm{H}, \mathrm{H}_{\mathrm{c}}\right), 7.58\left(\mathrm{~d}, 4 \mathrm{H}, \mathrm{H}_{\mathrm{b}}\right), 7.08\left(\mathrm{~s}, 4 \mathrm{H}, \mathrm{H}_{\mathrm{a}}\right), 4.35\left(\mathrm{t}, 4 \mathrm{H}, \mathrm{H}_{\mathrm{d}}\right), 3.47\left(\mathrm{t}, 4 \mathrm{H}, \mathrm{H}_{\mathrm{g}}\right), 3.39(\mathrm{~m}$, $\left.32 \mathrm{H}, \mathrm{H}_{\mathrm{h}}\right), 1.85\left(\mathrm{td}, 4 \mathrm{H}, \mathrm{H}_{\mathrm{e}}\right), 1.72\left(\mathrm{td}, 4 \mathrm{H}, \mathrm{H}_{\mathrm{f}}\right)$ and $1.49\left(\mathrm{~m}, 32 \mathrm{H}, \mathrm{H}_{\mathrm{i}}\right)$.<smiles>CC(OC(=O)c1ccc(N2C(=O)C=CC2=O)cc1)C(I)C(I)COC(C)(C)C(C)CCOC(=O)c1ccc(N2C(=O)C=CC2=O)cc1</smiles>

\subsubsection{Synthesis of aliphatic maleimide derivatives}

In a 3-neck round bottomed flask, equipped with a dropping funnel, maleic anhydride (2.1 eq) was dissolved in THF (with an anhydride concentration of $2 \mathrm{~mL} \cdot \mathrm{g}^{-1}$ ). Diamine (1 eq) was added dropwise via a dropping funnel and stirred $2 \mathrm{~h}$ at room temperature. After degassing under $\mathrm{N}_{2}$ during $20 \mathrm{~min}$, $\mathrm{ZnBr}_{2}$ (2.3 eq) was added in one portion under inert atmosphere. When the solid was dissolved, the mixture was heated at $40^{\circ} \mathrm{C}$. Then the HMDS solubilized in THF $\left(1 \mathrm{~mL} \cdot \mathrm{mL}^{-1}\right)$, was added dropwise via a dropping funnel. The reaction mixture was stirred during $3 \mathrm{~h}$ at $70^{\circ} \mathrm{C}$. A viscous brown liquid was obtained. The product was extracted with water and washed three times with chloroform. After drying with $\mathrm{Na}_{2} \mathrm{SO}_{4}$ the solvent of the organic layer was evaporated. Lastly the product was dissolved in toluene $\left(3 \mathrm{~mL} \cdot \mathrm{g}^{-1}\right)$ and heated at $100^{\circ} \mathrm{C}$ overnight to obtain the maleimide form[30].

$B M A l E D-600(x+y=3.6$ and $z=9)$ (A). Orange viscous liquid, yield 64\%. ${ }^{1} \mathrm{H}$ NMR (400 MHz, $\left.\mathrm{CDCl}_{3}, \delta \mathrm{ppm}\right): 6.62\left(\mathrm{~s}, 4 \mathrm{H}, \mathrm{H}_{\mathrm{a}}\right), 4.34\left(\mathrm{~m}, 2 \mathrm{H}, \mathrm{H}_{\mathrm{c}}\right), 3.87-3.26\left(\mathrm{~m}, 48 \mathrm{H}, \mathrm{H}_{\mathrm{d}}, \mathrm{H}_{\mathrm{e}}, \mathrm{H}_{\mathrm{g}}\right.$ and $\left.\mathrm{H}_{\mathrm{h}}\right), 1.31-1.29$ (dd, $\left.6 \mathrm{H}, \mathrm{H}_{\mathrm{b}}\right), 1.11-0.98\left(\mathrm{~m}, 8 \mathrm{H}, \mathrm{H}_{\mathrm{f}}\right)$. The maleimide (1 eq) was mixed with furfuryl acetate $(2.1 \mathrm{eq})$ and stirred during 7 days at room temperature. This red and viscous product was stored in a freezer at $4{ }^{\circ} \mathrm{C}$ to avoid the evolution of endo/exo ratio.

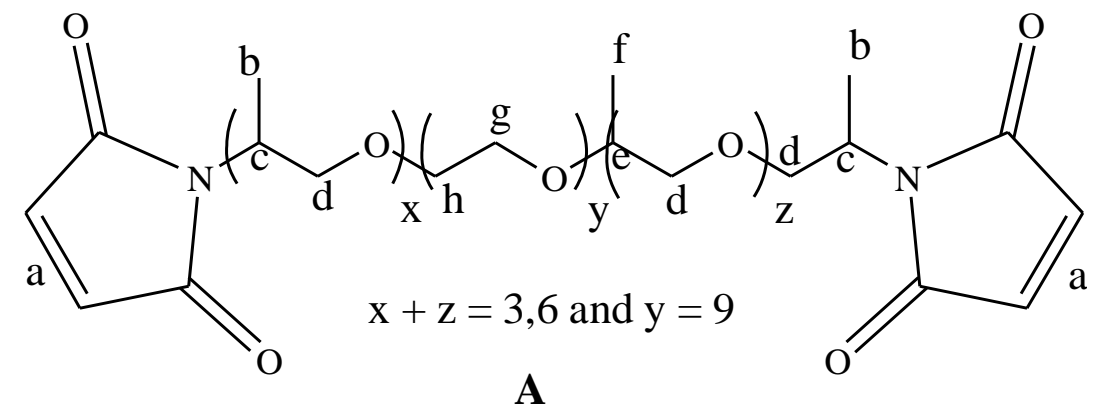

BM Al ED-600 BLQ furfuryl acetate $(x+y=3.6$ and $z=9)(\mathbf{B}) .{ }^{1} \mathrm{H} \mathrm{NMR}\left(400 \mathrm{MHz},\left(\mathrm{CD}_{3}\right)_{2} \mathrm{CO}\right.$, endo, $\delta$ ppm): 6.63-6.35 (m, 4H, Ha and Hb), 5.25 (d, 2H, 3Jcd = 5.5 Hz, Hc), 4.82-4.80 (ddd, 4H, Hf), 
4.13 (m, 2H, Hh), 3.9-3.24 (m, 56H, Hk, Hl, Hn, Ho, Hj,Hd et He), 2.03 (s, 6H, Hg), 1.31-0.98 (m, $11 \mathrm{H}$, Hi et $\mathrm{Hm}) .{ }^{1} \mathrm{H}$ NMR (400 MHz, $\left(\mathrm{CD}_{3}\right)_{2} \mathrm{CO}$, exo, $\left.\delta \mathrm{ppm}\right): 6.63-6.35$ (m, 4H, Ha and $\mathrm{Hb}$ ), 5.15 (d, $2 \mathrm{H}, 3 \mathrm{Jcd}=1.4 \mathrm{~Hz}, \mathrm{Hc}$ ), 4.85-4.34 (ddd, 4H, Hf), 4.29 (m, 2H, Hh), 3.9-3.24 (m, 52H, Hk, Hl, Hn, Ho and $\mathrm{Hj}$ ), 3.08-2.95 (m, 4H, He and $\mathrm{Hd}$ ), 2.06 (s, 6H, Hg), 1.31-0.98 (m, 11H, Hi et Hm).

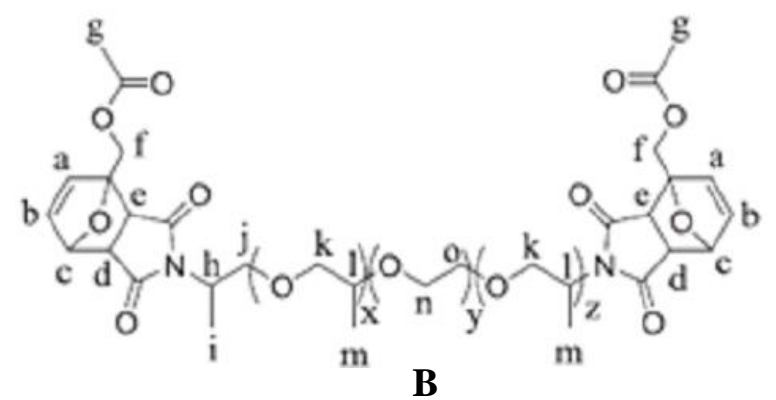

\subsubsection{Synthesis of blocked aromatic maleimide}

The maleimide ( 1 eq) was mixed with furfuryl acetate (2.1 eq) and stirred during 7 days at room temperature. This red and viscous product was stored in a freezer at $4{ }^{\circ} \mathrm{C}$ to avoid the evolution of endo/exo ratio.

$B M$ Ar L35 BLQ furfuryl acetate $(x+y=16$ and $z=21)$ (SI5). Red viscous liquid, yield $93 \% .{ }^{1} \mathrm{H}$ NMR (400 MHz, $\left(\mathrm{CD}_{3}\right)_{2} \mathrm{CO}$, endo, $\left.\delta \mathrm{ppm}\right): 8.12-8.1\left(\mathrm{~m}, 4 \mathrm{H}, \mathrm{H}_{\mathrm{i}}\right), 7.38-7.36\left(\mathrm{~m}, 4 \mathrm{H}, \mathrm{H}_{\mathrm{h}}\right), 6.72-6.71(\mathrm{~d}$, $\left.2 \mathrm{H}, \mathrm{H}_{\mathrm{a}}\right), 6.6\left(\mathrm{t}, 2 \mathrm{H}, \mathrm{H}_{\mathrm{b}}\right), 5.42-5.41\left(\mathrm{dd}, 2 \mathrm{H}, \mathrm{H}_{\mathrm{c}}\right), 4.91-4.57\left(\mathrm{ddd}, 4 \mathrm{H}, \mathrm{H}_{\mathrm{f}}\right), 4.48\left(\mathrm{t}, 4 \mathrm{H}, \mathrm{H}_{\mathrm{j}}\right), 3.92(\mathrm{td}, 2 \mathrm{H}$, $\mathrm{H}_{\mathrm{d}}$ ), 3.85 (td, 4H, $\mathrm{H}_{\mathrm{k}}$ ), 3.69-3.34 (m, 172H, $\mathrm{H}_{\mathrm{e}}, \mathrm{H}_{\mathrm{l}}, \mathrm{H}_{\mathrm{m}}, \mathrm{H}_{\mathrm{n}}, \mathrm{H}_{\mathrm{o}}$ ), $2.08\left(\mathrm{~s}, 6 \mathrm{H}, \mathrm{H}_{\mathrm{g}}\right), 1.11\left(\mathrm{~m}, 62 \mathrm{H}, \mathrm{H}_{\mathrm{p}}\right){ }^{1}$ HNMR (400 MHz, $\left(\mathrm{CD}_{3}\right)_{2} \mathrm{CO}$, exo, $\left.\delta \mathrm{ppm}\right): 8.16-8.14\left(\mathrm{~m}, 4 \mathrm{H}, \mathrm{H}_{\mathrm{i}}\right), 7.50-7.48\left(\mathrm{~m}, 4 \mathrm{H}, \mathrm{H}_{\mathrm{h}}\right), 6.72-6.71(\mathrm{~d}$, $\left.2 \mathrm{H}, \mathrm{H}_{\mathrm{a}}\right), 6.6\left(\mathrm{t}, 2 \mathrm{H}, \mathrm{H}_{\mathrm{b}}\right), 5.31\left(\mathrm{~s}, 2 \mathrm{H}, \mathrm{H}_{\mathrm{c}}\right), 4.87-4.50\left(\mathrm{ddd}, 4 \mathrm{H}, \mathrm{H}_{\mathrm{f}}\right), 4.48\left(\mathrm{t}, 4 \mathrm{H}, \mathrm{H}_{\mathrm{j}}\right), 3.85\left(\mathrm{td}, 4 \mathrm{H}, \mathrm{H}_{\mathrm{k}}\right.$ ), 3.69-3.34 (m, 172H, $\left.\mathrm{H}_{\mathrm{e}}, \mathrm{H}_{\mathrm{l}}, \mathrm{H}_{\mathrm{m}}, \mathrm{H}_{\mathrm{n}}, \mathrm{H}_{\mathrm{o}}\right), 2.04\left(\mathrm{~s}, 6 \mathrm{H}, \mathrm{H}_{\mathrm{g}}\right), 1.11$ (m, 62H, $\mathrm{H}_{\mathrm{p}}$ ).

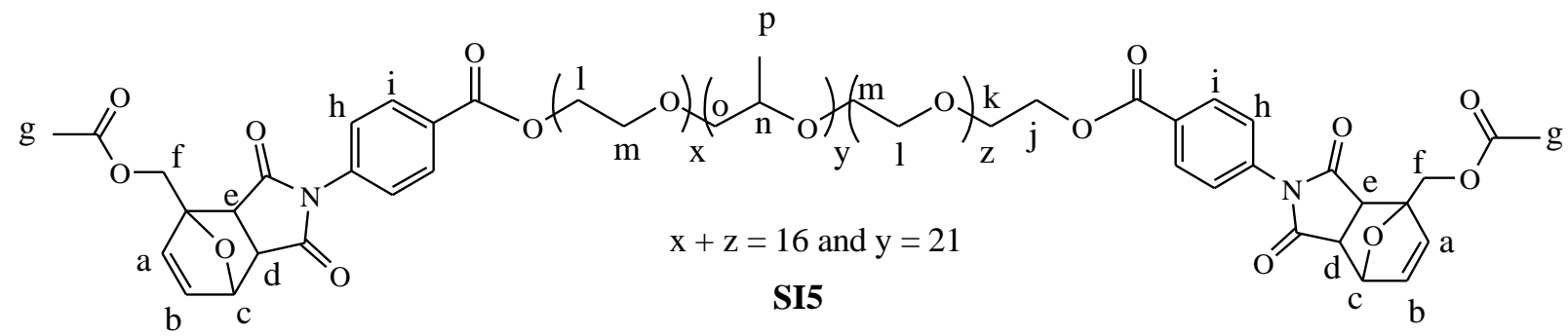

BM Ar ED-600 BLQ furfuryl acetate $\left(x+y=3.6\right.$ and $z=9$ ) (SI6). Red viscous liquid, yield $94 \% .{ }^{1} \mathrm{H}$ NMR (400 MHz, $\left(\mathrm{CD}_{3}\right)_{2} \mathrm{CO}$, endo, $\left.\delta \mathrm{ppm}\right): 7.94-7.91\left(\mathrm{~m}, 4 \mathrm{H}, \mathrm{H}_{\mathrm{i}}\right), 7.59-7.47\left(\mathrm{~s}_{\mathrm{br}}, 2 \mathrm{H}, \mathrm{H}_{\mathrm{j}}\right), 7.26-7.24(\mathrm{~m}$, $\left.4 \mathrm{H}, \mathrm{H}_{\mathrm{h}}\right), 6.7-6.68\left(\mathrm{~d}, 2 \mathrm{H}, \mathrm{H}_{\mathrm{a}}\right), 6.57\left(\mathrm{t}, 2 \mathrm{H}, \mathrm{H}_{\mathrm{b}}\right), 5.39-5.38\left(\mathrm{dd}, 2 \mathrm{H}, \mathrm{H}_{\mathrm{c}}\right), 4.89-4.56\left(\mathrm{ddd}, 4 \mathrm{H}, \mathrm{H}_{\mathrm{f}}\right), 4.27$ (s, $\left.2 \mathrm{H}, \mathrm{H}_{\mathrm{j}}\right), 3.88\left(\mathrm{td}, 2 \mathrm{H}, \mathrm{H}_{\mathrm{d}}\right), 3.65-3.64\left(\mathrm{~d}, 2 \mathrm{H}, \mathrm{H}_{\mathrm{e}}\right), 3.55\left(\mathrm{~m}, 48 \mathrm{H}, \mathrm{H}_{\mathrm{m}}, \mathrm{H}_{\mathrm{n}}, \mathrm{H}_{\mathrm{o}}, \mathrm{H}_{\mathrm{p}}\right), 2.07\left(\mathrm{~s}, 6 \mathrm{H}, \mathrm{H}_{\mathrm{g}}\right), 1.26$ and $1.09\left(2 \mathrm{~m}, 12 \mathrm{H}, \mathrm{H}_{\mathrm{k}}\right) .{ }^{1} \mathrm{H} \mathrm{NMR}\left(400 \mathrm{MHz},\left(\mathrm{CD}_{3}\right)_{2} \mathrm{CO}\right.$, exo, $\left.\delta \mathrm{ppm}\right): 7.98-7.96\left(\mathrm{~m}, 4 \mathrm{H}, \mathrm{H}_{\mathrm{i}}\right), 7.59-7.47$ $\left(\mathrm{s}_{\mathrm{br}}, 2 \mathrm{H}, \mathrm{H}_{\mathrm{j}}\right), 7.38-7.36\left(\mathrm{~m}, 4 \mathrm{H}, \mathrm{H}_{\mathrm{h}}\right), 6.7-6.68\left(\mathrm{~d}, 2 \mathrm{H}, \mathrm{H}_{\mathrm{a}}\right), 6.57\left(\mathrm{t}, 2 \mathrm{H}, \mathrm{H}_{\mathrm{b}}\right), 5.29\left(\mathrm{dd}, 2 \mathrm{H}, \mathrm{H}_{\mathrm{c}}\right), 4.86-4.47$ (ddd, 4H, $\mathrm{H}_{\mathrm{f}}$ ), $4.27\left(\mathrm{~s}, 2 \mathrm{H}, \mathrm{H}_{\mathrm{j}}\right), 3.55\left(\mathrm{~m}, 48 \mathrm{H}, \mathrm{H}_{\mathrm{m}}, \mathrm{H}_{\mathrm{n}}, \mathrm{H}_{\mathrm{o}}, \mathrm{H}_{\mathrm{p}}\right), 3.30-3.20\left(\mathrm{dd}, 2 \mathrm{H}, \mathrm{H}_{\mathrm{d}}, \mathrm{H}_{\mathrm{e}}\right), 2.02(\mathrm{~s}, 6 \mathrm{H}$, $\left.\mathrm{H}_{\mathrm{g}}\right), 1.26$ and $1.09\left(2 \mathrm{~m}, 12 \mathrm{H}, \mathrm{H}_{\mathrm{k}}\right)$. 


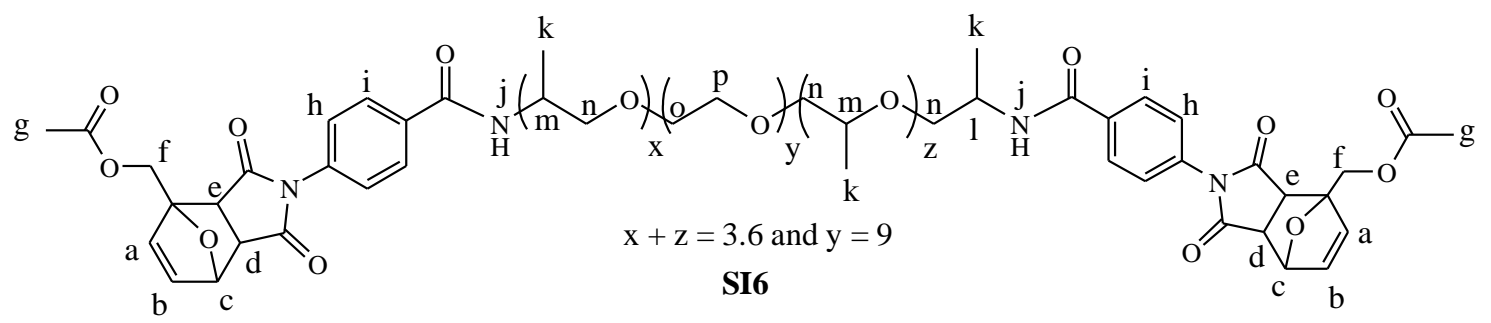

BM Ar ED-900 BLQ Furfuryl Acetate $(x+y=6$ and $z=12.5)$ (SI7). Red viscous liquid, yield 89\%. ${ }^{1} \mathrm{H}$ NMR $\left(400 \mathrm{MHz},\left(\mathrm{CD}_{3}\right)_{2} \mathrm{CO}\right.$, endo, $\left.\delta \mathrm{ppm}\right)$ : 7.94-7.92 (m, 4H, $\left.\mathrm{H}_{\mathrm{i}}\right), 7.61-7.42\left(\mathrm{~s}_{\mathrm{br}}, 2 \mathrm{H}, \mathrm{H}_{\mathrm{j}}\right), 7.26-7.24$ $\left(\mathrm{m}, 4 \mathrm{H}, \mathrm{H}_{\mathrm{h}}\right), 6.7-6.68\left(\mathrm{~d}, 2 \mathrm{H}, \mathrm{H}_{\mathrm{a}}\right), 6.57\left(\mathrm{t}, 2 \mathrm{H}, \mathrm{H}_{\mathrm{b}}\right), 5.39-5.38\left(\mathrm{dd}, 2 \mathrm{H}, \mathrm{H}_{\mathrm{c}}\right), 4.86-4.56\left(\mathrm{ddd}, 4 \mathrm{H}, \mathrm{H}_{\mathrm{f}}\right), 4.28$ (s, 2H, $\mathrm{H}_{\mathrm{j}}$ ), $3.88\left(\mathrm{td}, 2 \mathrm{H}, \mathrm{H}_{\mathrm{d}}\right), 3.65-3.64\left(\mathrm{~d}, 2 \mathrm{H}, \mathrm{H}_{\mathrm{e}}\right), 3.55\left(\mathrm{~m}, 76 \mathrm{H}, \mathrm{H}_{\mathrm{m}}, \mathrm{H}_{\mathrm{n}}, \mathrm{H}_{\mathrm{o}}, \mathrm{H}_{\mathrm{p}}\right), 2.07$ (s, 6H, $\mathrm{H}_{\mathrm{g}}$ ), 1.26 and 1.09 (2m, 16H, $\left.\mathrm{H}_{\mathrm{k}}\right) .{ }^{1} \mathrm{H}$ NMR (400 MHz, $\left(\mathrm{CD}_{3}\right)_{2} \mathrm{CO}$, exo, $\left.\delta \mathrm{ppm}\right)$ : 7.99-7.97 (m, 4H, $\mathrm{H}_{\mathrm{i}}$ ), 7.61-7.42 ( $\left.\mathrm{s}_{\mathrm{br}}, 2 \mathrm{H}, \mathrm{H}_{\mathrm{j}}\right), 7.38-7.36\left(\mathrm{~m}, 4 \mathrm{H}, \mathrm{H}_{\mathrm{h}}\right), 6.7-6.68\left(\mathrm{~d}, 2 \mathrm{H}, \mathrm{H}_{\mathrm{a}}\right), 6.57\left(\mathrm{t}, 2 \mathrm{H}, \mathrm{H}_{\mathrm{b}}\right), 5.29\left(\mathrm{dd}, 2 \mathrm{H}, \mathrm{H}_{\mathrm{c}}\right)$, 4.89-4.48 (ddd, 4H, $\left.\mathrm{H}_{\mathrm{f}}\right), 4.28\left(\mathrm{~s}, 2 \mathrm{H}, \mathrm{H}_{\mathrm{j}}\right), 3.55\left(\mathrm{~m}, 76 \mathrm{H}, \mathrm{H}_{\mathrm{m}}, \mathrm{H}_{\mathrm{n}}, \mathrm{H}_{\mathrm{o}}, \mathrm{H}_{\mathrm{p}}\right), 3.30-3.20\left(\mathrm{dd}, 2 \mathrm{H}, \mathrm{H}_{\mathrm{d}}, \mathrm{H}_{\mathrm{e}}\right.$ ), $2.02\left(\mathrm{~s}, 6 \mathrm{H}, \mathrm{H}_{\mathrm{g}}\right), 1.24$ and $1.09\left(2 \mathrm{~m}, 16 \mathrm{H}, \mathrm{H}_{\mathrm{k}}\right)$.

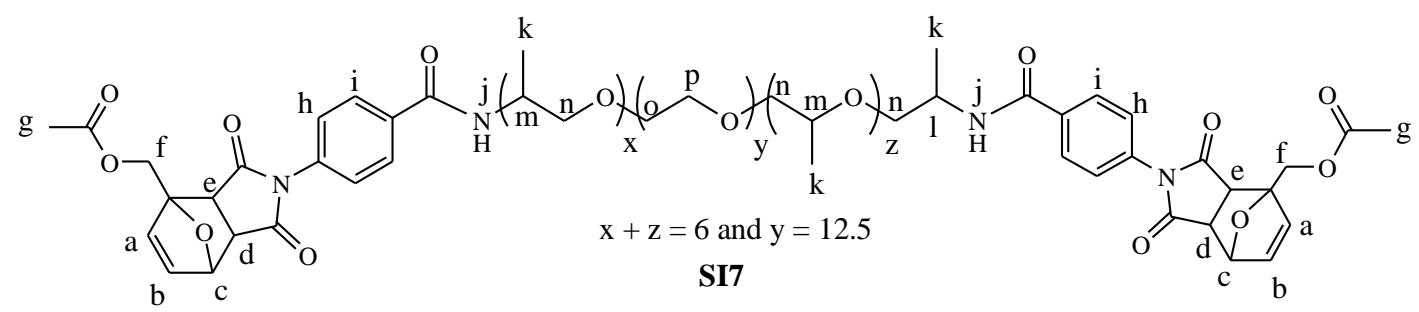

BM Ar Terat650 BLQ furfuryl acetate $(y=8)$ (SI8). Red viscous liquid, yield 93\%. ${ }^{1} \mathrm{H}$ NMR (400 $\mathrm{MHz},\left(\mathrm{CD}_{3}\right)_{2} \mathrm{CO}$, endo, $\left.\delta \mathrm{ppm}\right): 8.09-8.07\left(\mathrm{~m}, 4 \mathrm{H}, \mathrm{H}_{\mathrm{i}}\right), 7.35-7.33\left(\mathrm{~m}, 4 \mathrm{H}, \mathrm{H}_{\mathrm{h}}\right), 6.7-6.69\left(\mathrm{~d}, 2 \mathrm{H}, \mathrm{H}_{\mathrm{a}}\right)$, $6.58\left(\mathrm{t}, 2 \mathrm{H}, \mathrm{H}_{\mathrm{b}}\right), 5.4-5.39\left(\mathrm{dd}, 2 \mathrm{H}, \mathrm{H}_{\mathrm{c}}\right), 4.87-4.56\left(\mathrm{ddd}, 4 \mathrm{H}, \mathrm{H}_{\mathrm{f}}\right), 4.36\left(\mathrm{td}, 4 \mathrm{H}, \mathrm{H}_{\mathrm{j}}\right), 3.89\left(\mathrm{td}, 2 \mathrm{H}, \mathrm{H}_{\mathrm{d}}\right)$, 3.67-3.66 (d, 2H, $\left.\mathrm{H}_{\mathrm{e}}\right), 3.47\left(\mathrm{~m}, 32 \mathrm{H}, \mathrm{H}_{\mathrm{m}}\right), 3.39\left(\mathrm{~m}, 32 \mathrm{H}, \mathrm{H}_{\mathrm{n}}\right), 2.07\left(\mathrm{~s}, 6 \mathrm{H}, \mathrm{H}_{\mathrm{g}}\right), 1.86\left(\mathrm{~m}, 4 \mathrm{H}, \mathrm{H}_{\mathrm{k}}\right), 1.71$ $\left(\mathrm{m}, 4 \mathrm{H}, \mathrm{H}_{\mathrm{l}}\right), 1.59\left(\mathrm{~m}, 32 \mathrm{H}, \mathrm{H}_{\mathrm{o}}\right) .{ }^{1} \mathrm{H} \mathrm{NMR}\left(400 \mathrm{MHz},\left(\mathrm{CD}_{3}\right)_{2} \mathrm{CO}\right.$, exo, $\left.\delta \mathrm{ppm}\right): 8.13-8.12\left(\mathrm{~m}, 4 \mathrm{H}, \mathrm{H}_{\mathrm{i}}\right)$, 7.47-7.46 (m, 4H, $\left.\mathrm{H}_{\mathrm{h}}\right), 6.7-6.69\left(\mathrm{~d}, 2 \mathrm{H}, \mathrm{H}_{\mathrm{a}}\right), 6.58\left(\mathrm{t}, 2 \mathrm{H}, \mathrm{H}_{\mathrm{b}}\right), 5.29\left(\mathrm{dd}, 2 \mathrm{H}, \mathrm{H}_{\mathrm{c}}\right), 4.90-4.48\left(\mathrm{ddd}, 4 \mathrm{H}, \mathrm{H}_{\mathrm{f}}\right)$, $4.36\left(\mathrm{td}, 4 \mathrm{H}, \mathrm{H}_{\mathrm{j}}\right), 3.47\left(\mathrm{~m}, 4 \mathrm{H}, \mathrm{H}_{\mathrm{m}}\right), 3.39\left(\mathrm{~m}, 32 \mathrm{H}, \mathrm{H}_{\mathrm{n}}\right), 3.32-3.23\left(\mathrm{dd}, 2 \mathrm{H}, \mathrm{H}_{\mathrm{d}}, \mathrm{H}_{\mathrm{e}}\right), 2.01\left(\mathrm{~s}, 6 \mathrm{H}, \mathrm{H}_{\mathrm{g}}\right), 1.86$ $\left(\mathrm{m}, 4 \mathrm{H}, \mathrm{H}_{\mathrm{k}}\right), 1.71\left(\mathrm{~m}, 4 \mathrm{H}, \mathrm{H}_{\mathrm{l}}\right), 1.59\left(\mathrm{~m}, 32 \mathrm{H}, \mathrm{H}_{\mathrm{o}}\right)$.

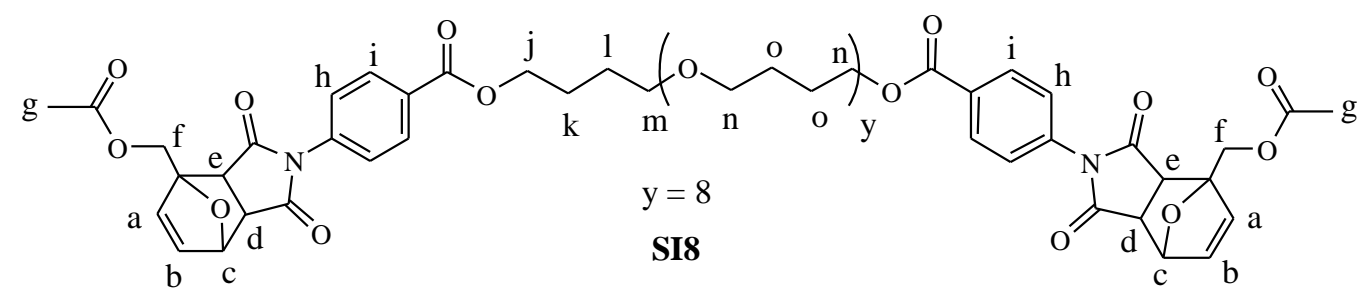

\subsubsection{Synthesis of polymers thermosets}

Bismaleimides (1 eq.) and 2-mercaptoethyl ether (ME) (1 eq.) were mixed and left for $48 \mathrm{~h}$ at room temperature and then they were post-cured at $150^{\circ} \mathrm{C}$ for $24 \mathrm{~h}$. Standard procedure for kinetic experiments: The kinetic experiments were performed in a NMR tube in DMSO- $d_{6}$ at $70^{\circ} \mathrm{C}$ and with a ratio of 1:1 between maleimide function and thiophenol. The reaction was monitored by ${ }^{1} \mathrm{H}$ NMR spectroscopy with the disappearance of the methylene of the ester (the aromatic protons of the thiophenol were used as an internal standard. A ${ }^{1} \mathrm{H}$ NMR experiment was performed every 15 minutes. 


\section{Results and discussion}

\subsection{Synthesis of blocked aromatic maleimides}

The aromatic bis-maleimides were synthesized following the adapted procedure of Fache et al. which consists first to synthesize the maleimide from 4-aminobenzoic acid (Scheme 1) [34]. The pure 4-maleimidobenzoic acid was obtained in good yield (88\%). Then, the two-step synthesis of telechelic oligomers was performed. In the first step, $\mathrm{SOCl}_{2}$ was used to functionalize the acid into acyl chloride function (yield 73\%). The second step consisted in an amidification or an esterification reaction with respectively diols or diamines in quantitative yield. Thus, we have synthesized a new aromatic liquid polymeric bismaleimide from $\mathrm{SOCl}_{2}$, a less toxic reactant than $\mathrm{COCl}_{2}$.

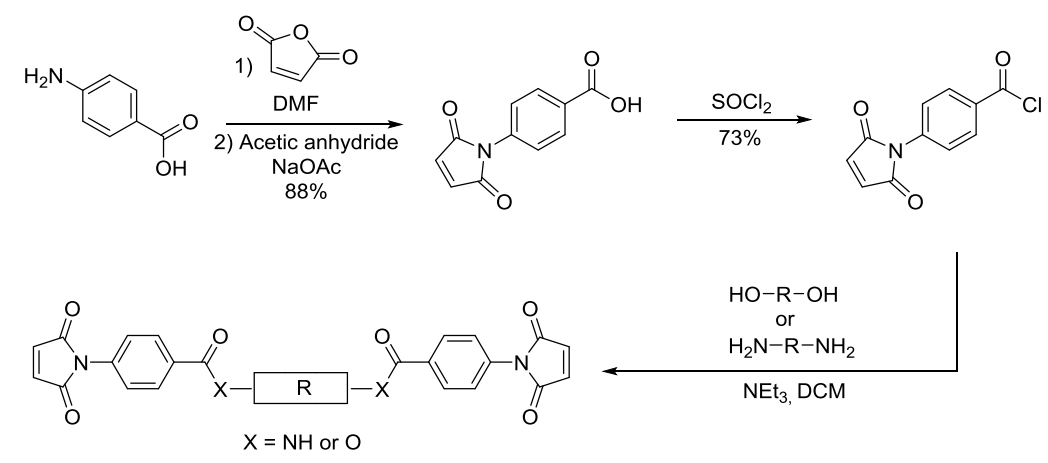

Scheme 1 Synthesis route to obtain aromatic polymeric bismaleimides.

The aliphatic bismaleimide was previously synthesized [30]. The different maleimides synthesized in this article will be referred as follows: $\mathrm{B}$ for bis $(f=2)$, followed by $\mathrm{M}$ for maleimide, $\mathrm{Ar}$ in case of aromatic maleimide or $\mathrm{Al}$ in case of aliphatic maleimide respectively and finally the spacer name. The characteristics of polymeric bismaleimides are presented in Table 1.

Table 1 summary of characteristics of synthesized polymeric bismaleimides

\begin{tabular}{|c|c|c|c|c|}
\hline Hardener & $\mathrm{Y}$ & $\mathrm{x}+\mathrm{z}$ & Physical form & Yield* $1 \%$ \\
\hline BM Al ED-600 & 9 & 3.6 & Liq. & 64 \\
\hline BM Ar ED-600 & 9 & 3.6 & Liq. & 63 \\
\hline BM Ar ED-900 & 12.5 & 6 & Liq. & 62 \\
\hline BM Ar L35 & 16 & 21 & Liq. & 56 \\
\hline $\begin{array}{l}\text { zBM } \\
\text { Terat-650 }\end{array}$ & 9 & l & $\operatorname{Liq}\left(T>25^{\circ} \mathrm{C}\right)$ & 60 \\
\hline
\end{tabular}

* Overall yield.

All bismaleimides were synthesized with good overall yields and are liquid except for the BM $\mathrm{Ar}$ Terat- 650 which is liquid only above $25^{\circ} \mathrm{C}$. These bismaleimides have been used for the study of the DA reaction.

\subsection{Study of DAl}

It is already known that during the DA reaction, two diastereomers are formed: endo and exo diastereomers. The ratio endo:exo varies depending on several factors such as the temperature of the DA reaction or the electronic environment. Thus, in a recent work [26], we have studied in detail this DA 
reaction on model molecules according to the electronic environment with the aim of determining the substituent groups that favor the rDA reaction at low temperature. As a conclusion, the more this substituent is electro-withdrawing, the more the diastereisomer exo is formed.

A same study was performed with the polymeric bismaleimides previously synthesized which were compared to the aliphatic bismaleimides. Hence, the polymeric bismaleimides and furfuryl acetate were mixed at room temperature for 7 days. The protection rates and the ratios endo/exo were determined by ${ }^{1} \mathrm{H}$ NMR. The protection rates of the aromatic maleimides were similar, around $90 \%$. The endo diastereoisomer was more favored when the aliphatic maleimide was used (Table 2). Furthermore, the presence of ester or amide groups in bismaleimide did not had a strong influence on the exo/endo ratio.

Table 2 Protection rates and endo/exo adducts ratios for each liquid maleimide blocked with furfuryl acetate at room temperature

\begin{tabular}{lcccc}
\hline Maleimide & Functions of spacer & Protection rate $/ \%$ & \multicolumn{2}{c}{ Diastereomers $/ \%$} \\
\hline BM Al ED-600 & Ether & 95 & 80 & 20 \\
BM Ar ED-600 & Amide & 94 & 67 & 33 \\
BM Ar ED-900 & Amide & 89 & 54 & 46 \\
BM Ar L35 & Ester & 93 & 51 & 49 \\
BM Ar Terat-650 & Ester & 93 & 46 & 54 \\
\hline
\end{tabular}

\subsection{Study of rDA reaction}

\subsubsection{Model reactions}

Previous studies showed that rDA reactions were neither be influenced by the diastereoisomer endo/exo ratio nor the reactants or products concentrations [26]. However, the presence of a thiol in the reaction modifies the kinetic of this rDA reaction. Indeed, the thiol-Michael addition reaction onto the maleimide is very fast and competes with the isomerization reaction of the endo into exo diastereoisomer (thermodynamically diastereoisomer).

In order to compare the reactivity of both aromatic and aliphatic polymeric bismaleimides, only the polymeric bismaleimides prepared from diamines of Huntsman, Jeffamine ${ }^{\circledR}$, were used and their retro-DA reaction was monitored by ${ }^{1} \mathrm{H}$ NMR. Thus, in order to avoid the isomerization of the endo diasteroisomer into exo diastereoisomer during the rDA, thiophenol was added to the mixture, prior heating at $70{ }^{\circ} \mathrm{C}$.

First, model maleimides (methyl maleimide and $p$-nitro maleimide) were synthesized to study the rDA at $70{ }^{\circ} \mathrm{C}$ in presence of thiophenol without any catalyst. Thanks to the high reactivity of aromatic maleimide, the endo diastereoisomer of this compound was fully deprotected, faster than the endo diastereoisomer of the aliphatic moiety (Fig. 1). Furthermore, the kinetic experiments have shown that at $70{ }^{\circ} \mathrm{C}$, the exo diastereoisomer of the aliphatic maleimide stayed protected. Indeed, aliphatic bismaleimides were deprotected at higher temperature than aromatic one. 


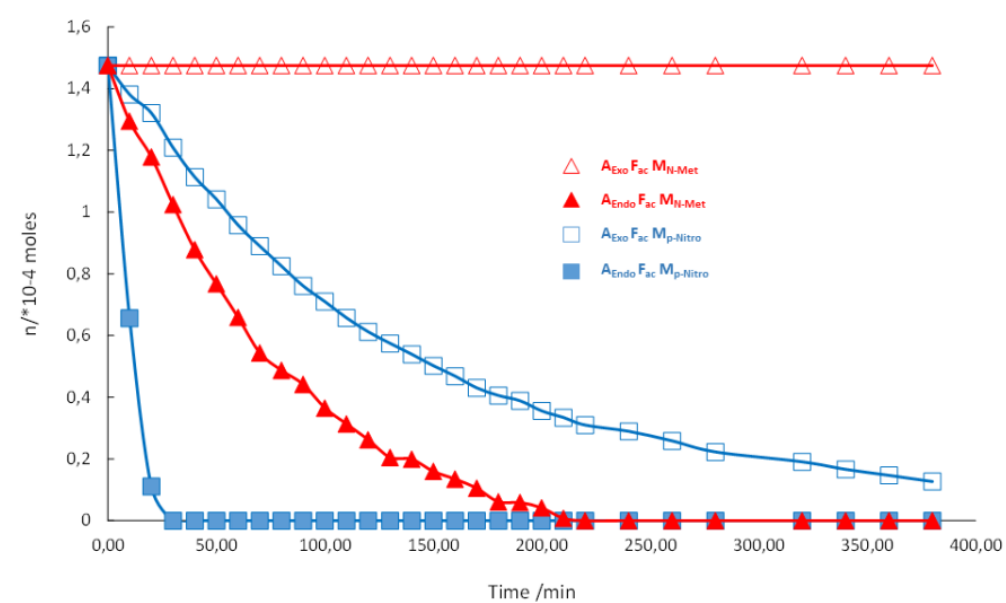

Fig. 1 Comparison of deprotection reaction kinetics of diastereomers endo/exo of $N$-methylmaleimide $\left(\mathrm{M}_{\mathrm{N}-\mathrm{Met}}\right) /$ furfuryl acetate $\left(\mathrm{F}_{\mathrm{ac}}\right)$ and $p$-nitrophenylmaleimide $\left(\mathrm{M}_{\mathrm{p} \text {-Nitro }}\right) /$ furfuryl acetate adducts in presence of thiophenol at $70{ }^{\circ} \mathrm{C}$

\subsubsection{Bis-maleimides kinetic reactions}

Then, the reactivities of the aromatic and aliphatic polymeric bismaleimides were compared using ${ }^{1} \mathrm{H}$ NMR kinetic experiments (Fig. 2) (SI9; SI10). As for the model molecules, the endo diastereoisomer of the aromatic maleimide was deprotected faster than the aliphatic one. After 150 minutes, the endo aromatic diastereoisomer was fully deprotected, whereas the deprotection of the aliphatic one required about 400 minutes. However, the exo diastereoisomer of the aromatic bismaleimide behaved differently from the model molecule. Indeed, the exo diastereoisomer of the aromatic bismaleimide stayed partially protected at $70{ }^{\circ} \mathrm{C}$ (whereas it was consumed when the model aromatic maleimide was used. Indeed, the aromatic bismaleimide bears a less electro withdrawing group (ester group) than the model molecule (nitro group). Hence, the Hammett substituent constant is around 0.40 for an amide in para-position whereas it is 0.78 for a nitro in para-position [35] Thus, the reactivity of the aromatic-bismaleimide is far different from the one of the model molecule.

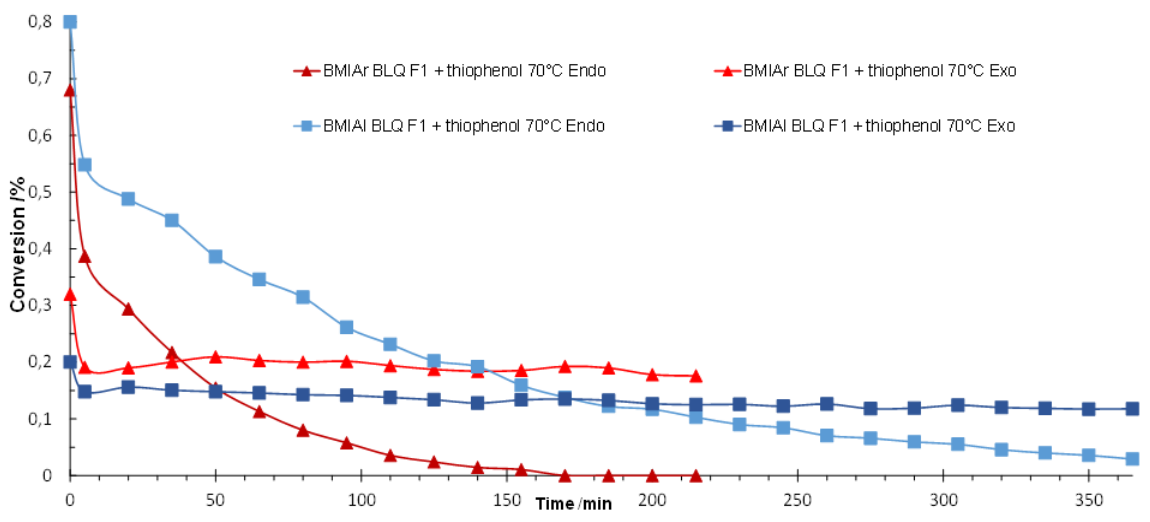

Fig. 2 Comparison of deprotection reaction kinetics of diastereomers endo/exo of BM Al/furfuryl acetate and BM Ar/furfuryl acetate adducts in presence of thiophenol at $70{ }^{\circ} \mathrm{C}$ 


\subsection{Synthesis and characterization of polymers}

The two bismaleimides have been reacted with bis(2-mercaptoethyl) ether (ratio1:1) to compare the influence of aliphatic and aromatic bismaleimides on thermal properties. These mixtures were polymerized at room temperature for $48 \mathrm{~h}$ and heated at $150{ }^{\circ} \mathrm{C}$ to achieve the polymerization.

Low glass transition temperatures were obtained for these polymers due to the high flexibility conferred by the PPO and PEO chains (SI11; SI12). However, as expected, the presence of aromatic moieties in the BMI-Ar gave more rigidity to the system and thus the aromatic thermoset exhibited a higher $T_{g}$ value ( 14 vs. $-20^{\circ} \mathrm{C}$ ) (Table 3 ).

Table 3 Thermal properties of the polymers

\begin{tabular}{cccc}
\hline Materials & $T_{\mathrm{g}} /{ }^{\circ} \mathrm{C}$ & $\mathrm{T}_{\mathrm{d} 5 \%} * /{ }^{\circ} \mathrm{C}$ & Char yield $/ \%$ \\
\hline BMI-Al + 2-MEE & -20 & 304 & 12 \\
BMI-Ar + 2-MEE & 14 & 358 & 25 \\
\hline
\end{tabular}

*degradation temperature at $5 \%$ weight loss

The thermal stability of these polymers was evaluated by TGA between room temperature and $700{ }^{\circ} \mathrm{C}$. All the materials have a degradation temperature at $5 \%$ weight loss above $300{ }^{\circ} \mathrm{C}$. As expected, the presence of aromatic moieties permitted to double char yield (Table 3).

\section{Conclusions}

This study describes a novel cross-linking "on demand" system using aromatic bismaleimides. Thus, new aromatic bis-functional blocked maleimides with ester or amide bonds were synthesized and their DA reactivity were compared to the one of aliphatic maleimides. The DA reaction of aromatic maleimides gave higher contents of exo diastereomer than the aliphatic ones. Regarding the retro-DA reaction, the reactivity of the endo aromatic diastereomer was higher than the aliphatic bismaleimide one. After 150 min reaction, the aromatic endo adduct was completely consumed, whereas the consumption of exo diastereomer was far lower. Then, polymers were synthesized to compare their thermal properties. As expected, the aromatic bismaleimide monomers conferred higher rigidity to the materials which exhibited higher glass transition and degradation temperatures and higher char content than the aliphatic bismaleimide-based polymers.

Acknowledgments The authors thank the Hutchinson Company for its financial support, Biobased Polymer Company for reactants supply.

\section{References}

1. Zhang Y, Broekhuis A A, Picchioni F. Thermally self-healing polymeric materials: The next step to recycling thermoset polymers? Macromolecules, 2009, 42(6): 1906-1912 doi:10.1021/ma8027672

2. Gandini A. The application of the Diels-Alder reaction to polymer syntheses based on furan/maleimide reversible couplings. Polímeros, 2005, 15(2): 95-101 doi:10.1590/S0104-14282005000200007

3. Keller M W, Pascault J P, Williams R J J. Self-Healing epoxy composites. In: Epoxy Polymers. Weinheim, Germany: Wiley-VCH Verlag GmbH \& Co. KGaA, 2010, 325-344 
4. Wicks D A, Wicks Z W Jr. Blocked isocyanates. III: Part A. Mechanisms and chemistry. Progress in Organic Coatings, 1999, 36(3): 148-172 doi:10.1016/S0300-9440(99)00042-9

5. Pazos J F. Polymers coupled by nitroso groups. US Patent, 3872057A, 1975-05-18

6. Montarnal D, Capelot M, Tournilhac F, Leibler L. Silica-like malleable materials from permanent organic networks. Science, 2011, 334(6058): 965-968 doi:10.1126/science.1212648

7. Gordon J. Additions and corrections-fused organic salts. III. Chemical stability of molten tetra-n-alkylammonium salts. Medium effects on thermal $\mathrm{R}_{4} \mathrm{~N}^{+} \mathrm{X}^{-}$-decomposition. $\mathrm{RBr}+\mathrm{I}^{-}=\mathrm{RI}+\mathrm{Br}^{-}$equilibrium constant in fused salt medium. Journal of Organic Chemistry, 1965, 30(12): 4396 doi:10.1021/jo01023a634

8. Ko E C F, Leffek K T, 0. Studies on the decomposition of tetra-alkylammonium salts in solution. V. Rates and activation parameters of salts containing substituted-benzyl or other active groups. Canadian Journal of Chemistry, 1972, 50(9): 1297-1302 doi:10.1139/v72-203

9. Leffek K T, Matheson A F. Secondary kinetic isotope effects in bimolecular nucleophilic substitutions. VI. Effect of $\alpha$ and $\beta$ deuteration of alkyl halides in their Menschutkin reactions with pyridine in nitrobenzene. Canadian Journal of Chemistry, 1972, 50(7): 986-991 doi:10.1139/v72-155

10. Wagener K B, Engle L P, Woodard M H. Thermally reversible polymer linkages. 1. Model studies of the azlactone ring. Macromolecules, 1991, 24(6): 1225-1230 doi:10.1021/ma00006a001

11. Wagener K B, Engle L P. Thermally reversible polymer linkages. 3. Covalently crosslinked poly(azlactone). Macromolecules, 1991, 24(26): 6809-6815 doi:10.1021/ma00026a001

12. Gheneim R, Perez-Berumen C, Gandini A. Diels-Alder reactions with novel polymeric dienes and dienophiles: Synthesis of reversibly cross-linked elastomers. Macromolecules, 2002, 35(19): 7246-7253 doi:10.1021/ma020343c

13. Diels O, Alder K. Synthesen in der hydroaromatischen Reihe. Justus Liebigs Annalen der Chemie, 1928, 460(1): 98-122 doi:10.1002/jlac. 19284600106

14. Gandini A. The furan/maleimide Diels-Alder reaction: A versatile click-unclick tool in macromolecular synthesis. Progress in Polymer Science, 2013, 38(1): 1-29 doi:10.1016/j.progpolymsci.2012.04.002

15. Bailey W F, Wachter-Jurcsak N M, Pineau M R, Ovaska T V, Warren R R, Lewis C E. Acetylenic vinyllithiums: Consecutive cycloisomerization- [4 + 2] cycloaddition reactions. Journal of Organic Chemistry, 1996, 61(23): 8216-8228 doi:10.1021/jo9614371

16. Roush W R, Sciotti R J. Enantioselective total synthesis of (-)-chlorothricolide via the tandem inter- and intramolecular Diels-Alder reaction of a hexaenoate intermediate. Journal of the American Chemical Society, 1998, 120(30): 7411-7419 doi:10.1021/ja980611f

17. Javadi A, Mehr H S, Sobani M, Soucek M D. Cure-on-command technology: A review of the current state of the art. Progress in Organic Coatings, 2016, 100: 2-31 doi:10.1016/j.porgcoat.2016.02.014

18. Ishizu K, Makino M, Hatoyama N, Uchida S. Emulsion-induced ordered microporous films using amphiphilic poly(ethylene oxide)-block-poly(n-butyl isocyanate) block copolymers. Journal of Applied Polymer Science, 2008, 108(6): 3753-3759 doi:10.1002/app.28030

19. Ji Y, Kim J, Bae J Y. Flame-retardant ABS resins from novel phenyl isocyanate blocked novolac phenols and triphenyl phosphate. Journal of Applied Polymer Science, 2006, 102(1): 721-728 doi:10.1002/app.23258 
20. Gironès J, Pimenta M T B, Vilaseca F, Carvalho A J F, Mutjé P, Curvelo A A S. Blocked diisocyanates as reactive coupling agents: Application to pine fiber-polypropylene composites. Carbohydrate Polymers, 2008, 74(1): 106-113 doi:10.1016/j.carbpol.2008.01.026

21. Sankar G, Nasar A S. Cure-reaction kinetics of amine-blocked polyisocyanates with alcohol using hot-stage fourier transform infrared spectroscopy. Journal of Applied Polymer Science, 2008, 109(2): 1168-1176 doi:10.1002/app.28172

22. Moszner N, Zeuner F, Salz U, Rheinberger V. Reaction behaviour of monomeric B-Ketoesters. Polymer Bulletin, 1994, 33(1): 43-49 doi:10.1007/BF00313472

23. Viganò M, Levi M, Turri S, Chiari M, Damin F. New copolymers of $N, N$-dimethylacrylamide with blocked isocyanates for oligonucleotide immobilization in DNA microarray technology. Polymer, 2007, 48(14): 4055-4062 doi:10.1016/j.polymer.2007.05.019

24. Ubaghs L, Keul H, Höcker H. Novel intramolecular blocked isocyanates as stable one-component systems for poly(urea urethane)s. Polymer, 2005, 46(5): 1459-1465 doi:10.1016/j.polymer.2004.12.028

25. Lou C, Gu J, Di M, Ma L, Wang Y, Liu X. Synthesis and characterization of trichlorophenol-blocked polyaryl polyisocyanate. Iranian Polymer Journal, 2011, 20(3): 247-255

26. Froidevaux V, Borne M, Laborbe E, Auvergne R, Gandini A, Boutevin B. Study of the Diels-Alder and retro-Diels-Alder reaction between furan derivatives and maleimide for the creation of new materials. RSC Advances, 2015, 5(47): 37742-37754 doi:10.1039/C5RA01185J

27. Canadell J, Fischer H, De With G, van Benthem R A T M. Stereoisomeric effects in thermo-remendable polymer networks based on Diels-Alder crosslink reactions. Journal of Polymer Science. Part A, Polymer Chemistry, 2010, 48(15): 3456-3467 doi:10.1002/pola.24134

28. Chan J W, Hoyle C E, Lowe A B, Bowman M. Nucleophile-initiated thiol-Michael reactions: Effect of organocatalyst, thiol, and ene. Macromolecules, 2010, 43(15): 6381-6388 doi:10.1021/ma101069c

29. Billiet S, Van Camp W, Hillewaere X K D, Rahier H, Du Prez F E. Development of optimized autonomous self-healing systems for epoxy materials based on maleimide chemistry. Polymer, 2012, 53(12): 2320-2326 doi:10.1016/j.polymer.2012.03.061

30. Froidevaux V, Negrell C, Laborbe E, Auvergne R, Boutevin B. Thermosetting material by a thermo responsive cross-linking using retroDiels-Alder and, in Situ, thia-Michael reactions. European Polymer Journal, 2015, 69: 510-522 doi:10.1016/j.eurpolymj.2015.03.044

31. Dolci E, Froidevaux V, Joly-Duhamel C, Auvergne R, Boutevin B, Caillol S. Maleimides as a building block for the synthesis of high performance polymers. Polymer Reviews (Philadelphia, Pa.), 2016, 56(3): 512-556 doi:10.1080/15583724.2015.1116094

32. Herr D, Sridhar L, Slark A. Thermally reversible hot melt adhesive composition containing multifunctional diene and dienophile compounds. US Patent, 20120082840 A1, 2012-05-04

33. Fache B, Gallot B, Gelin M P, Milano J C, Pham Q T. Synthesis and thermal properties of bismaleimides with mesogen aromatic amide-ester and flexible polymethylenic group. Journal of Applied Polymer Science, 2013, 127(5): 3798-3813 doi:10.1002/app.37659

34. Fache B, Mekkid S, Milano J C, Vernet J L. Synthèse et tenue thermique de bisnadimides et bismaléimides avec groupement souple de type polyéthylène glycol. European Polymer Journal, 1998, 34(11): 1621-1627 doi:10.1016/S0014-3057(98)00020-2 
35. Hansch C, Leo A, Taft R W. A survey of Hammett substituent constants and resonance and field parameters. Chemical Reviews, 1991, 91(2): 165-195 doi:10.1021/cr00002a004 